\title{
A FLAIR FOR MULTI-OBJECT SPECTROSCOPY
}

\author{
Q.A. PARKER ${ }^{1}$ and F.G. WATSON ${ }^{2}$ \\ ${ }^{1}$ Anglo-Australian Observatory, PO Box 296, Epping NSW 2121, Australia \\ ${ }^{2}$ Royal Greenwich Observatory, Madingley Road, Cambridge, U.K.
}

SUMMARY. Unique in the regime of wide-field astronomy is the multi-object spectroscopy system 'FLAIR' developed for the UK Schmidt Telescope (UKST). The current system can perform simultaneous observations of $\sim 90$ objects across 40 sq. degrees of sky. FLAIR has given a whole new platform for performing astronomical observations with a Schmidt telescope in a non-photographic role. The system is simple to operate, highly stable, easy to interchange with standard photography and outclasses singleobject spectroscopy systems on $2.0 \mathrm{~m}$ class telescopes for many types of observation. The system has continued to be developed to enhance its flexibility, robustness and versatility, culminating recently in the commissioning of new fibre feeds and formats. The facility to observe independent standard stars, and the capability of observing objects in two UKST fields in a single night has recently been demonstrated. FLAIR is a powerful, cost-effective addition to the instrumental repertoire of the Anglo-Australian Observatory in a way that was not even remotely envisaged when the UKST was built.

\section{The FLAIR System}

FLAIR, an acronym for Fibre Linked Array Image Re-formatter, is the only facility in the world for performing multi-object spectroscopy (MOS) of moderately faint objects $(B \sim 17-18)$ across a wide field. The system works by relaying light from the focal surface of the UKST via lowloss optical fibres within a modified photographic plateholder to a fast, highly efficient spectrograph on the dome floor.

Because of the fine plate scale of the UKST the fibres need to be positioned to an accuracy of $\sim 10 \mu \mathrm{m}(\sim 0.7 \mathrm{arcsec})$ in order to intercept fully the light from each target object. Rather than using punched aperture plates, FLAIR utilises the information on the photographic plates themselves to obtain the necessary accuracy. Optical fibres terminated with $2 \mathrm{~mm}$ square right angle prisms are simply cemented directly over the target images on a copy plate of the field of interest using a UV curing optical adhesive. This is achieved using an accurate, semi-automatic, $\mathrm{X}-\mathrm{Y}-\mathrm{Z}$ positioning system based on a $\mathrm{PC}$ controlled robotic gripper.

Various prototypes were developed (e.g. Watson 1986, 1988) until the current, fully engineered system known as FLAIR-II was commissioned in March 1992 (Watson et al. 1993). This system yielded a five-fold gain in observing efficiency and the great potential of the telescope began to be realised. Since FLAIR-II commissioning, the 100 nights allocated to the system have produced $\sim 6500$ observations of galaxies, stars, planetary nebulae and quasars. 
Observations were obtained for $\sim 83 \%$ of the allotted time (a higher figure than for photographic plates as only spectroscopic conditions are required). Both photography and FLAIR observations can be performed during the same night with the change over typically taking $\sim 20$ minutes.

The system's inherent mechanical stability comes from use of a floor mounted spectrograph. Appropriately this uses a Schmidt type collimator and camera to combine wide angular field with fast focal ratio. The gratings are the same as those used by the RGO spectrograph and planned for the 2 degree field (2df) fibre instrument of the AAT. Spectral resolutions from 1.4 to 12 Angstroms are provided. The excellent stability enables a minimum of arc exposures and other overheads thus maximising field dwell time.

Two Plateholders (PHs) are currently available. The first has $92 \times 100 \mu \mathrm{m}(6.7$ arcsec) fibres and $5 \times 33 \mu \mathrm{m}(2.2$ arcsec) fiducial-star fibres used for field acquisition and guiding. The newly commissioned 2nd PH is a 'hybrid' containing 152 individual fibres split between 55 and $100 \mu \mathrm{m}$ fibre formats. There are $73 \times 100 \mu \mathrm{m}(6.7 \mathrm{arcsec})$ fibres and $71 \times 55 \mu \mathrm{m}(3.7 \mathrm{arcsec})$ fibres, together with $8 \times 33 \mu \mathrm{m}(2.2 \mathrm{arcsec})$ fiducial fibres. The system's overall flexibility has thus been substantially improved because the $55 \mu \mathrm{m}$ fibres are better optimised to observations of point sources as the contribution from the sky-background light is reduced. However atmospheric dispersion does begin to play an increasing role at zenith distances $>45$ degrees with the smaller diameter fibres. The retention of a separate $100 \mu \mathrm{m}$ fibre bundle in the same PH offers increased flexibility, opportunities for carrying out different projects with the same PH on the same night and the possibility of 144 individual observations by swapping over fibre bundles halfway through a night. The ability to observe two fields during the long winter lunations has recently been demonstrated and both PHs can be prepared well in advance.

\section{Standard Object Observations}

Until recently, one of the major drawbacks of FLAIR was that observations of standard stars was not supported unless such a standard happened to be present in the target UKST field. However a scheme to circumvent this problem has recently been developed which makes use of the acquisition image-guide, a dedicated 'standard' fibre and the excellent pointing/tracking of the Schmidt. Standard stars which can be situated in any part of the sky have now been observed with little extra telescope overheads ( 40 minutes for two bright standards including $5-10$ minutes exposure times). This is now offered as an extra capability of FLAIR-II.

\section{Suitable Projects}

FLAIR can benefit many projects that can make efficient use of the wide field capability and multiplex advantage. It is particularly suited to objects of intermediate brightness $(B \leq 18.0)$ and a relatively sparse distribution on the sky ( 1 - 10 object per square degree). It is thus complementary with the soon to be commissioned 2df MOS system on the AAT as they fulfil different roles in terms of practical magnitude limit and area coverage.

Galaxy redshift surveys to $B \leq 17.5$ have been a major area of use (e.g. Parker \& Watson 1990; Watson et al. 1991; Broadbent et al. 1992). Planetary nebulae, emission line objects and quasars have also been successfully observed. Various stellar observations are also becoming increasingly common (e.g. Morgan, Watson \& Parker 1992). With the introduction of the second PH with an option for smaller diameter fibres, fainter point source observations can also be made. 

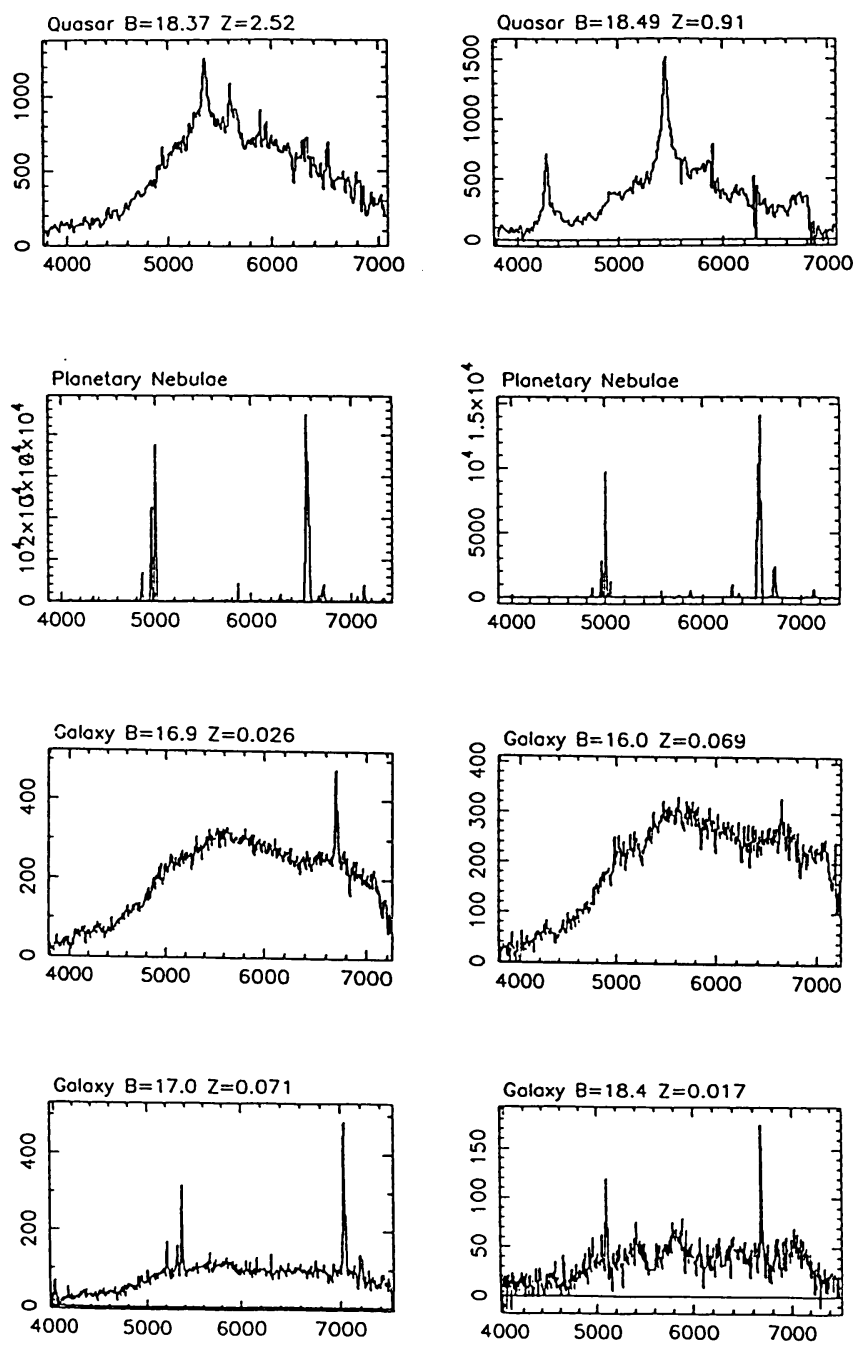

Figure 1. A Montage of FLAIR-II spectra from various object types.

The montage gives 8 spectra for 4 different FLAIR projects undertaken during 1992. The first 12 spectra are new quasars identified for a large-area UVX quasar survey from Lance Miller and Pippa Goldschmidt (Royal Observatory Edinburgh) sampling to $B=18.5$. The total combined exposure time was $\sim 7$ hours. The next two spectra are examples of planetary nebulae in the Galactic Bulge for a PN abundances project for Mike Barlow, Nic Walton and R. Clegg (UCL). The PN typically have $-14.5<\log F(H \beta)<-12.5$ in c.g.s units and the spectra are for a total of 1.8 hours observation. The subsequent row illustrates a couple of galaxies observed by Michael Drinkwater et al. (AAO), in the Virgo cluster area as part of a search for compact dwarf galaxies sampling to $B=17.0$ (total exposure time 3.3 hours). The remaining two spectra are for faint galaxies observed by Brian Clarke and Paul Hewett (Cambridge) from a sample of AGN candidates selected from UKST prism plates and/or with UV excess. In this case the spectra come from a combined total exposure of 8.5 hours. 


\section{Conclusions}

FLAIR is a unique facility offering an extremely efficient means of performing multi-object observations over a wide field. During a 5 night 'run' with the system there is the potential to observe $\sim 750$ individual observations if both PHs are utilised. Despite the modest aperture of the Schmidt, the power of FLAIR comes from the exceedingly long field dwell times available (e.g. $9 \times 3000$ seconds exposure), the wide field coverage ( 40 sq. degrees), and the large multiplex advantage. FLAIR is thus an ideal survey instrument for many applications for objects brighter than $B \sim 17-18$ (the exact limiting magnitude depends on the target object properties and desired resolution) and number densities $>1-2$ per square degree.

During 1994 an upgrade of the current CCD detector to one with much enhanced blue sensitivity is envisaged which should further improve the system performance.

\section{References}

Broadbent, A., Hale-Sutton, D., Shanks, T., Watson, F.G., Oates, A.P., Fong, R., Collins, C.A., MacGillivray, H.T., Nichol, R. and Parker, Q.A., 1992. 'The Durham-UKST Galaxy Redshift Survey', in 'Digitised Optical Sky Surveys', eds. H.T. MacGillivray and E.B. Thomson, Kluwer Academic Publishers, Dordrecht, p. 389.

Morgan, D.H., Watson, F.G. and Parker, Q.A., 1992. 'Emission line objects in the Large Magellanic Cloud', Astron. Astrophys. Suppl. Ser., 93, 495.

Parker, Q.A. and Watson, F.G., 1990. 'Galaxy redshifts with FLAIR', Astron. Astrophys. Suppl. Ser., 84, 455.

Watson, F.G., 1986. 'A wide field multi-object spectroscopy (MOS) system', in 'Instrumentation in Astronomy VI', Proc. SPIE, 627, 787.

Watson, F.G., 1988. 'Multi-object spectroscopy with FLAIR', in 'Fiber Optics in Astronomy', A.S.P. Conference Series, 3, 125.

Watson, F.G., Oates,A.P., Shanks, T. and Hale-Sutton, D., 1991. 'CCD multi-object spectroscopy of galaxies at the UKST', Mon. Not. R. astron. Soc., 253, 222.

Watson, F.G., Gray, P.M., Oates, A.P. and Bedding, T.R., 1993. 'The FLAIR-II system at the UK Schmidt Telescope', in 'Optics in Astronomy', Cambridge University Press, in press. 